\title{
Suppression of allergic airway inflammation and IgE responses by a class I restricted allergen peptide vaccine
}

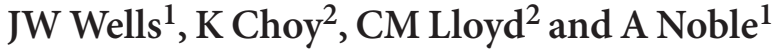

CD8 T cells are known to deviate CD4 T-cell responses from Th2 toward Th1. Reduction of Th2 cytokines and increased interferon- $\gamma$ ameliorates allergic airway disease. We have developed a novel approach to the suppression of allergic airway inflammation, by designing a $\mathrm{MHC}$ class I-restricted allergen peptide vaccine, which induces potent and longlived CD8 T-cell responses. Vaccination of C57BL/6 mice before allergen sensitization completely prevented allergenspecific immunoglobulin E (IgE) antibody responses. Vaccination after sensitization failed to suppress IgE, but inhibited accumulation of eosinophils and neutrophils in airways after subsequent allergen challenge. Vaccination suppressed Th2 airway infiltration and enhanced the lung Th1 response without inducing excessive CD8 cellular infiltration or interleukin-17, and the combination of class I peptide with adjuvant was more effective than adjuvant alone. Airway hyperreactivity was prevented by vaccination in an allergen-specific fashion. Class I peptide vaccines might therefore represent a robust and long-lasting immunotherapeutic strategy in allergic disease.

\section{INTRODUCTION}

Asthma is typically characterized by a persistent Th2-type CD4 T-cell infiltration of the lung tissue and airways, eosinophilia, mast cell infiltration of airway smooth muscle and high circulating immunoglobulin $\mathrm{E}$ (IgE). These allergic responses are primarily driven by CD $4 \mathrm{Th} 2$ cell responses to processed MHC class II-restricted epitopes derived from inhaled allergens. Allergen-specific CD4 T cells are required to provide the signals to B lymphocytes that drive them toward IgE synthesis, and directly mediate inflammatory pathology in the lung. Th1 cells counteract Th2 development and a deficient Th1 response to allergen is associated with atopy. ${ }^{1,2}$ Polymorphisms in CD14 and TLR2, molecules involved in activation of dendritic cells to induce Th1 responses, have been identified in children at high risk of atopy. ${ }^{3,4}$ Furthermore, allergic disease is usually initiated in early life, when competence for Th1 responses is at its lowest. ${ }^{5}$ The critical role played by the Th1 pathway in asthma prevention is indicated by mouse models, which show that genetic deletion of interferon- $\gamma($ IFN- $\gamma)$ results in enhanced allergic airway disease, ${ }^{6}$ overexpression of the Th1 transcription factor T-bet prevents airway remodeling, ${ }^{7}$ and virally induced, interleukin-17 (IL-17)-associated airway disease is accelerated in STAT1-deficient mice. ${ }^{8}$ In humans however, IFN- $\gamma$ treatment failed to improve steroid-dependent asthma despite inhibiting eosinophilia, ${ }^{9}$ whereas normal volunteers who inhaled nebulized IFN- $\gamma$ showed a slight improvement in lung function. ${ }^{10}$

It was recognized many years ago that $\mathrm{CD} 8 \mathrm{~T}$ cells are capable of inhibiting the IgE-associated responses to inhaled allergen. ${ }^{11}$ More recently the ability of allergen-specific, class I-restricted CD8 cells to suppress allergic airway inflammation and airway hyperreactivity has been demonstrated. ${ }^{12,13}$ This is attributed to collaboration between CD8 and CD4 T cells in invoking Th1type immunity. ${ }^{14,15}$ In humans, there is a negative correlation between number of CD8 cytotoxic cells in the blood and total IgE levels. ${ }^{16}$ To date however, no immunotherapeutics targeted to the class I:CD8 pathway have been developed, although successful anti-CD137 treatment is associated with CD8 cell expansion. ${ }^{17}$

Control of allergic symptoms with allergen immunotherapy has been used for many decades, but new approaches which avoid the potential for allergen-triggered, IgE-dependent side effects are needed. The use of MHC class II-restricted allergen-derived peptides has been successfully trialed in cat-sensitive asthma patients. ${ }^{18}$ This approach avoids the activation of

\footnotetext{
${ }^{1}$ King's College London, MRC and Asthma UK Centre in Allergic Mechanisms of Asthma, Guy's Hospital, London, UK. ${ }^{2}$ Imperial College London, MRC and Asthma UK Centre in Allergic Mechanisms of Asthma, National Heart and Lung Institute, London, UK. Correspondence: A Noble (alistair.noble@kcl.ac.uk) 
IgE-sensitized mast cells by whole allergen, but potentially activates preexisting Th2 effector cells. ${ }^{18}$ The Th1-polarizing adjuvant CpG DNA is effective in experimental asthma, but its effectiveness when given after sensitization may be short lived, because it can act via non-antigen-specific mechanisms. ${ }^{19}$ As Th1 memory is known to be short-lived in experimental models whereas CD8 memory is extremely long-lasting and existing adjuvants induce very poor CD8 T-cell responses, we developed a novel adjuvant combination capable of inducing potent and long-lived allergen-specific CD8 T-cell immunity. ${ }^{20}$ Here we have tested this novel anti-allergy vaccine, consisting of a class I peptide and a potent Th1-polarizing adjuvant and determined its ability to inhibit IgE responses, allergic airway inflammation and airway hyperreactivity in an experimental asthma model. The results show that targeting the CD8 $\mathrm{T}$-cell/Tc1 response is able to control allergic inflammation in the lung via immune deviation and might potentially provide a safer and more effective form of immunotherapy than whole allergen + Th1 adjuvant.

\section{RESULTS}

\section{Potent systemic and lung CD8 responses are induced by class I-restricted peptide and CASAC}

Responses to class I-restricted peptides are generally undetectable in the absence of adjuvants, and very weak when alum is used. We recently developed a novel adjuvant combination (CASAC) optimized for induction of CD8 OVA-specific responses in mice. ${ }^{20}$ We used this CASAC adjuvant, which results in much more potent CD8 responses than alternatives, in combination with SIINFEKL peptide in OVA-sensitized mice, to determine whether the CD8 T cells induced would migrate to allergen-sensitized lungs. Previous work had shown that responses to a class I peptide required either anti-CD40 antibody or a class II-restricted "helper peptide" to be added to the adjuvant. ${ }^{20}$ Using a version of CASAC containing anti-CD40 and SIINFEKL peptide, the CD8 T-cell responses to intradermal vaccination was greatest in blood (Figure 1a), and peaked at around 10 days after injection. Allergen-specific CD8 cells also accumulated in the spleen (Figure 1a). To assess lung CD8 responses, OVA/alum-sensitized mice were vaccinated with CASAC containing anti-CD40 and SIINFEKL peptide and 8 days later challenged with OVA intranasally on 6 consecutive days (Figure 1b). Accumulation of OVA-specific CD8 cells was seen in the lung tissue of sensitized mice given phosphatebuffered saline (PBS) in place of vaccine, however vaccination enhanced numbers of lung OVA-specific CD8 cells more than fourfold. As Tc2 type CD8 cells have been described in the lung, we tested the cytokine profile of vaccine-induced allergen-specific cells by restimulation of lung cells with SIINFEKL. Although poor antigen restimulation (typical of lung $\mathrm{T}$ cells in our hands) was observed, CD8 cells appeared preferentially to produce IFN- $\gamma$ rather than interleukin IL-4, suggesting they were Tc1-type (Figure 1c). Stimulation with a control peptide (Figure 1c left panel) resulted in background staining which was subtracted from the data to ensure that only OVA-specific cells were measured.
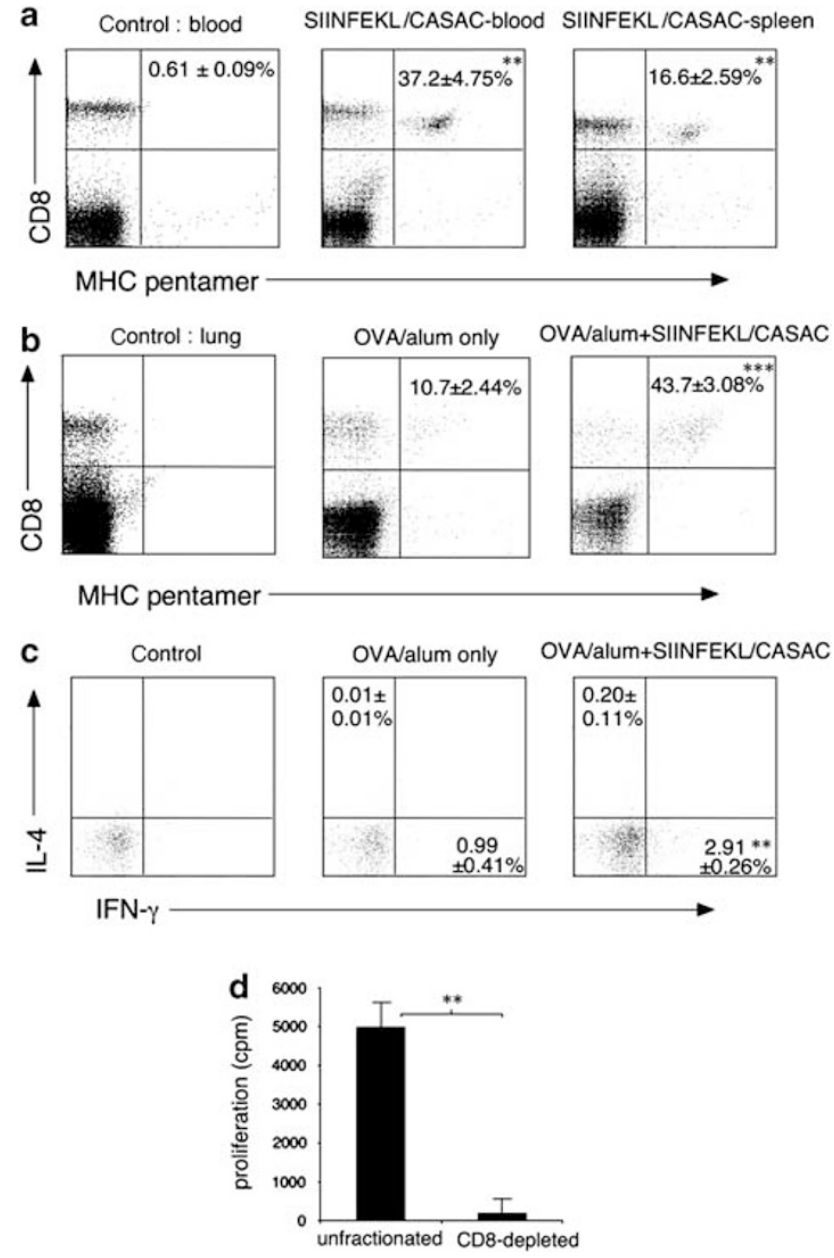

Figure 1 Class I peptide vaccination induces systemic and lung CD8 responses to allergen. (a) \% OVA-specific CD8 cells in blood and spleen 10 days after vaccination with SIINFEKL in CASAC + anti-CD40 adjuvant (SIINFEKL/CASAC) in OVA/alum-sensitized mice. ${ }^{* \star} P<0.005$ vs. control, $n=8$. (b) \% OVA-specific CD8 cells in lung tissue cells, with SIINFEKL/ CASAC/anti-CD40 vaccine (SIINFEKL/CASAC) or without vaccine (OVA/alum only), after intranasal allergen challenge. ${ }^{* \star \star} P<0.0005$ vs. OVA/alum alone, $n=6$. (c) Peptide-induced cytokine synthesis in CD8-gated lung cells, as in (b); background staining in controls has been subtracted, ${ }^{* \star} P<0.005, n=6$. (d) Proliferation of control and CD8depleted lymph node + spleen cells from SIINFEKL-vaccinated mice in response to SIINFEKL stimulation. Cells were cultured with or without peptide for 6 days; background proliferation in the absence of peptide has been subtracted, ${ }^{\star \star} P<0.005, n=3$.

To test whether responses to vaccination were purely CD8 Tcell mediated, we took pooled lymph node and spleen cells from SIINFEKL + CASAC immunized mice and stimulated them in vitro with SIINFEKL (Figure 1d). Cells were either unfractionated or depleted of CD 8 cells. This showed that $>95 \%$ of the SIINFEKL-specific proliferative response was CD8-mediated and the peptide vaccine did not therefore induce a CD4 T-cell response.

\section{Vaccination prevents primary allergic sensitization but not established IgE responses}

We assessed the effects of three distinct vaccines on $\operatorname{IgE}$ responses induced by $400 \mu \mathrm{g}$ OVA/alum (Figure 2). The 

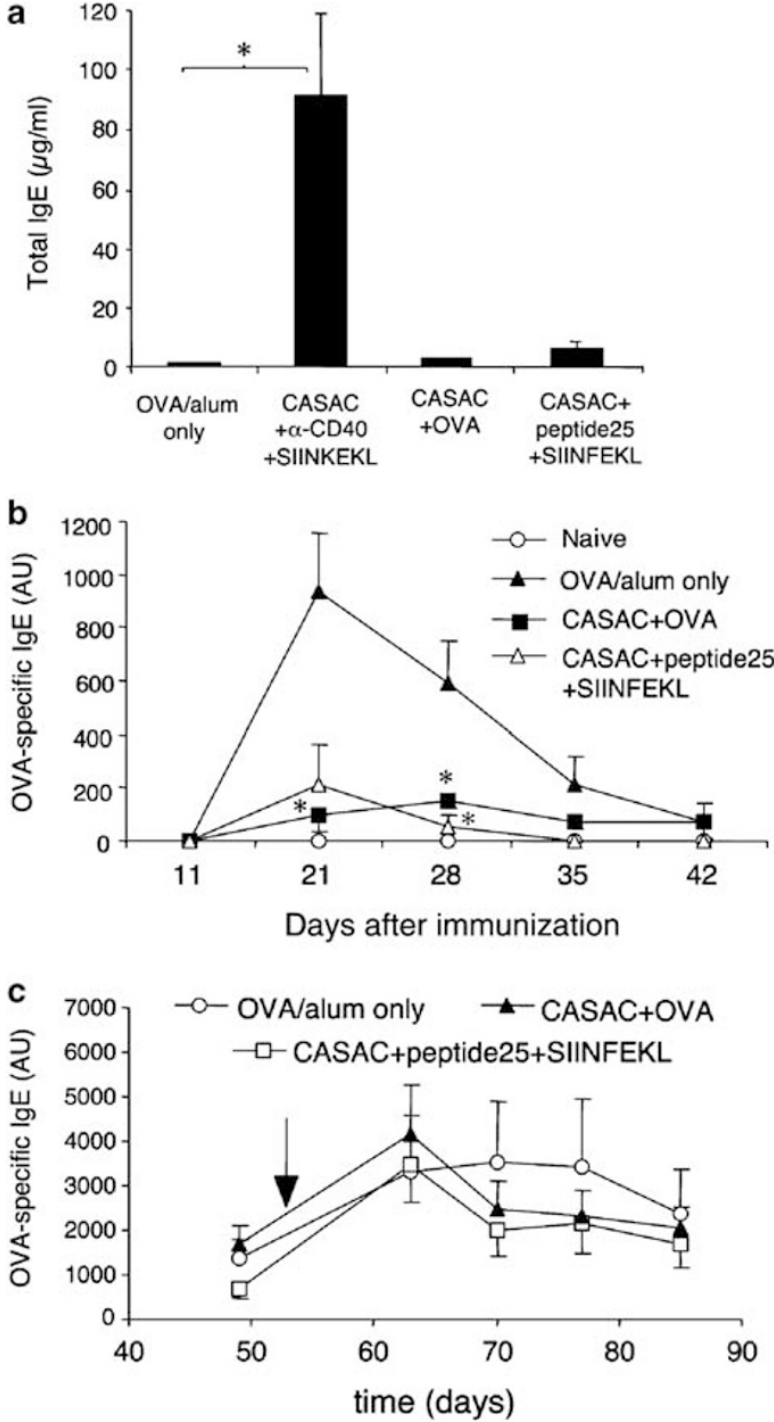

Figure 2 Effect of vaccination on immunoglobulin $\mathrm{E}$ (IgE) responses. (a) Effect of alternative adjuvant strategies on total serum IgE. Groups of 6 mice were immunized i.d. (OVA/Alum was given i.p.) with the vaccine combinations indicated on days 0 and 10; total serum IgE was measured by enzyme-linked immunosorbent assay (ELISA) on day 23. ${ }^{*} P<0.05$, $n=6$. (b) Nascent OVA-specific IgE production induced by OVA/alum after peptide or whole OVA protein vaccination (see Materials and Methods for details). Anti-CD40-containing vaccine did not suppress OVA-specific IgE and is omitted for clarity. ${ }^{*} P<0.05, n=6$. (c) Effect of vaccination on established long-term OVA-specific IgE levels induced by three OVA/alum injections. Mice were given a fourth and final boost of OVA/alum (indicated by arrow) following two rounds of peptide vaccination, (see Materials and Methods for details) $n=4$.

vaccine containing SIINFEKL and anti-CD40 in CASAC adjuvant was compared to one in which anti-CD40 was substituted with a class II "helper" peptide (peptide 25/p25) from an irrelevant antigen. Both strategies result in similar CD8 responses. ${ }^{20}$ The third vaccine omitted peptides and anti-CD40 and consisted of whole OVA + CASAC, resulting in both CD4 and substantial, but weaker CD8 OVA-specific responses. As shown in Figure 2a, two immunizations with the vaccine containing anti-CD40 was found to induce greatly elevated serum total IgE levels, how- ever, vaccines containing either helper peptide or whole allergen did not. The effect of these vaccines on allergen-specific IgE responses is shown in Figure 2b. Vaccination using either helper peptide, or whole OVA, before OVA/alum sensitization completely prevented the development of OVA-specific IgE responses, whereas vaccine containing anti-CD40 did not (not shown). When mice were repeatedly sensitized with OVA/alum to induce an ongoing IgE response and subsequently vaccinated, no significant suppression of OVA-specific IgE was observed (Figure 2c).

\section{Vaccination suppresses allergic airway inflammation after repeated allergen challenge}

As we have shown previously that allergen-specific CD8 T cells can suppress Th2-driven inflammation in the lung, ${ }^{13}$ we tested the hypothesis that inducing allergen-specific CD8 T cells with a vaccine would inhibit allergic airway disease. Mice were first sensitized with allergen in alum, then given a single dose of PBS or various vaccine combinations, and finally challenged with allergen intranasally (Table 1). A vaccine containing whole OVA was not used in these experiments as it would result in the undesired stimulation of allergen-specific CD4 cells. BAL T cells were prepared and intracellular cytokine production in CD4 $\mathrm{T}$ cells was compared after anti-CD3/CD28 stimulation. Both vaccine combinations resulted in reduced lung eosinophilia and decreased Th2 cells $(P<0.05)$. The level of IFN- $\gamma$ detectable in BAL fluid also significantly increased in response to both vaccine combinations. However, the anti-CD40 vaccine unexpectedly lead to a large increase in BAL IL-5 levels $(P<0.05, n=6$; Table 1), despite inhibiting IL-13 (data not shown). The impact of different vaccination strategies on airway hyperreactivity was assessed by resistance/compliance measurements after exposure to increasing doses of aerosolized methacholine. In mice not receiving vaccine, OVA sensitization and challenge resulted in dramatically increased sensitivity to methacholineinduced bronchoconstriction $(P<0.005$ vs. control at $30 \mathrm{mg} / \mathrm{ml}$ methacholine, $n=6$; Figure 3). Either vaccine (CASAC + antiCD40 + SIINFEKL or CASAC + p25 + SIINFEKL) effectively inhibited the development of airway hyperreactivity (both $P<0.05, n=6$; Figure 3), with responses in vaccinated mice being similar to baseline levels. However in light of the unexpected and undesired side effects of the anti-CD40 component on IgE and IL-5 levels, we performed subsequent experiments with the helper peptide vaccine.

\section{Allergen-specific CD8T cells are necessary for suppression of eosinophil infiltration}

To dissect the roles played by allergen-specific CD8-T cells and non-CD8-mediated effects of adjuvant components, we repeated the allergic airway inflammation experiments using an additional allergen (house dust mite (HDM)) and adjuvant-only control groups. These experiments used a lower allergen dose for sensitization than previously $(10 \mu \mathrm{g})$, because this dose is optimal for T-cell responses whereas high doses are optimal for IgE responses in C57BL/6 mice (not shown). Mice given CASAC alone or CASAC + p25 did not display a significant reduction in 
Table 1 Class I-restricted peptide vaccines inhibit allergic airway inflammation and skew BAL cytokine profiles

\begin{tabular}{|c|c|c|c|c|c|c|c|c|c|}
\hline & Allergen & Vaccine & $\begin{array}{l}\text { Total cells } \\
\left(\times 10^{-3}\right)\end{array}$ & $\begin{array}{l}\text { Eosinophils } \\
\left(\times 10^{-3}\right)\end{array}$ & $\begin{array}{c}\text { Neutrophils } \\
\left(\times 10^{-3}\right)\end{array}$ & $\%$ Th1 & \%Th2 & $\begin{array}{l}\text { IFN- } \gamma \\
(\mathrm{pg} / \mathrm{ml})\end{array}$ & IL-5 (pg/ml) \\
\hline 1 & PBS & PBS & $53.2 \pm 13.0$ & $3.5 \pm 1.1$ & $6.1 \pm 1.4$ & $6.6 \pm 0.5$ & $0.1 \pm 0.1$ & $19.9 \pm 1.7$ & $175 \pm 57.0$ \\
\hline 3 & OVA & $\begin{array}{l}\text { CASAC+Anti- } \\
\text { CD40+SIINFEKL }\end{array}$ & $171 \pm 38.0$ & $32.7 \pm 4.5^{\star}$ & $24.8 \pm 4.9$ & $6.5 \pm 0.6$ & $0.8 \pm 0.2^{*}$ & $186 \pm 36.5^{\star \star \star}$ & $1662 \pm 330^{*}$ \\
\hline 4 & OVA & $\begin{array}{l}\text { CASAC+peptide } \\
25+\text { SIINFEKL }\end{array}$ & $127 \pm 24.4$ & $35.5 \pm 6.2^{*}$ & $23.9 \pm 3.9$ & $14.2 \pm 2.1^{\star \star}$ & $0.8 \pm 0.2^{*}$ & $44.2 \pm 4.3^{*}$ & $560 \pm 145$ \\
\hline
\end{tabular}

IFN- $\gamma$, interferon- $\gamma$; IL-5, interleukin-5; PBS, phosphate-buffered saline.

Inflammatory cell numbers and cytokine profiles from BAL CD4 cells in mice sensitized (400 $\mu$ g OVA/alum) and challenged with OVA, and vaccinated with SIINFEKL peptide in CASAC adjuvant containing either anti-CD40 or an irrelevant class II helper peptide (peptide 25). ${ }^{*} \mathrm{P}<0.05,{ }^{* *} \mathrm{P}<0.01,{ }^{* * *} \mathrm{P}<0.001$, all in comparison to group 2. Results are from groups of 6 mice. Similar results were obtained in 3 independent experiments.

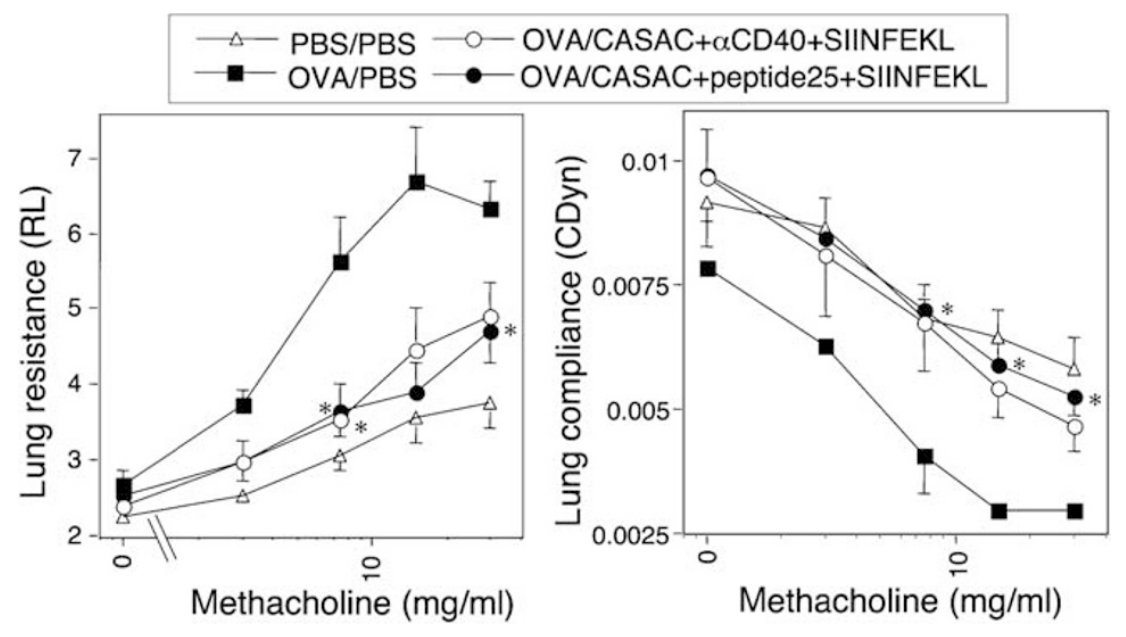

Figure 3 Airway hyperreactivity to methacholine challenge is abrogated by vaccination. Mice were sensitized to allergen ( $400 \mu \mathrm{g}$ OVA) using alum i.p. (days 0 and 12), given a single i.d. vaccine injection on Day 20, and then challenged i.n. with $50 \mu \mathrm{g}$ OVA daily on days 28-33. Lung resistance (left) and compliance (right) were measured on day 34 . ${ }^{*} P<0.05$ vs. OVA-sensitized (PBS) group $(n=6)$. Axis labels should be read "sensitizing allergen"/ "vaccine configuration".

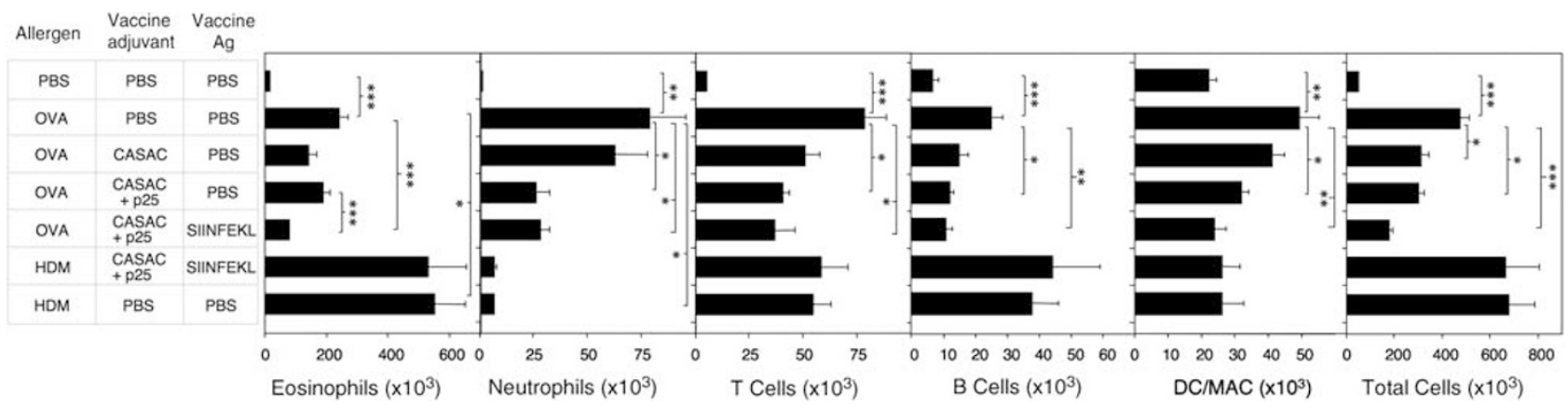

Figure 4 Peptide vaccination suppresses airway inflammation in response to inhaled OVA. Mice were sensitized with allergen (OVA or HDM, $10 \mu \mathrm{g})$ in alum (days 0 and 12) and injected i.d. with various vaccine combinations on day 20, then challenged i.n. with phosphate-buffered saline (PBS) or $50 \mu \mathrm{g}$ of the corresponding allergen daily on days $28-33$. Inflammatory cell infiltration in BAL fluid on day 34 was measured by flow cytometry. ${ }^{*}<0.05$, ${ }^{\star \star} P<0.005,{ }^{* * *} P<0.0005, n=6$. Similar results were obtained in three independent experiments.

infiltrating eosinophil numbers in response to intranasal OVA challenge (Figure 4). However, eosinophilia was significantly reduced by CASAC + p25 + SIINFEKL in OVA-sensitized animals $(P<0.0005, n=6)$ and SIINFEKL had a significant effect over adjuvant alone $(P<0.0005, n=6)$. Furthermore, eosinophil numbers were unaffected by CASAC + p $25+$ SIINFEKL vaccination in HDM-sensitized animals. Taken together, the data indicate that allergen-specific CD8 T cells are involved 
a

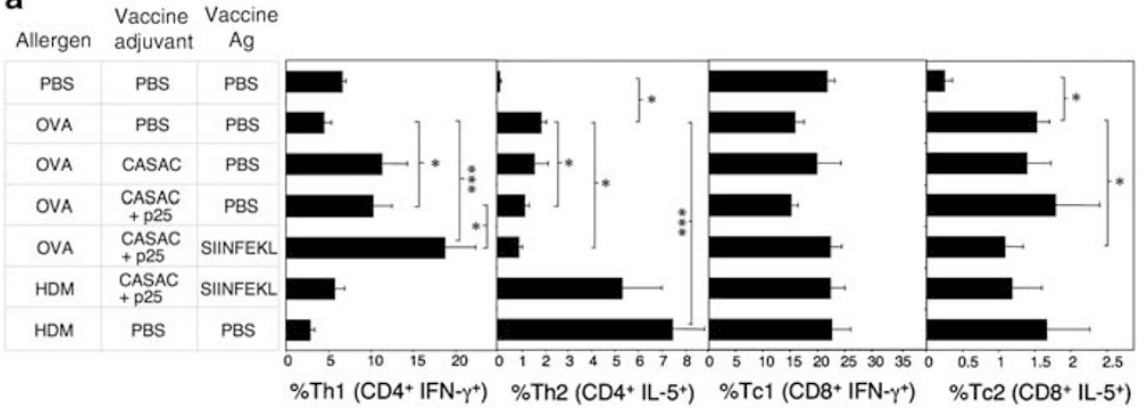

b

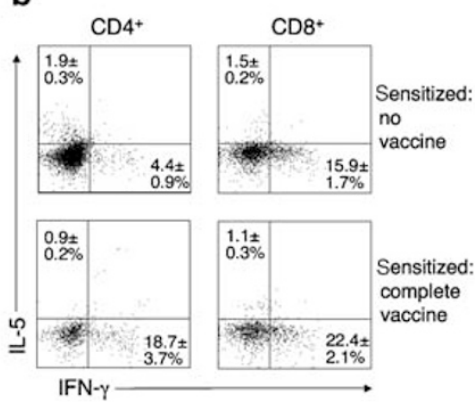

Figure 5 Peptide vaccination promotes immune deviation toward a Th1 CD4 response in the lung. (a) Mice were sensitized to allergen (10 $\mu$ g; days 0 and 12), given a single i.d. vaccine injection on day 20 , and challenged i.n. with the sensitizing allergen daily on days 28-33, as in Figure 4 . BAL T cells were stimulated with anti-CD3/28 and stained for intracellular interferon- $\gamma$ (IFN- $\gamma$; Th1/Tc1) and interleukin-5 (IL-5; Th2/Tc2). \% positive-staining cells in gated CD4 ${ }^{+}(\mathrm{Th})$ and $\mathrm{CD} 8{ }^{+}(\mathrm{Tc})$ populations are shown. ${ }^{*} P<0.05$, ${ }^{\star \star \star} P<0.0005, n=6$. (b) Representative flow cytometric cytokine staining profiles from gated CD4 and CD8 populations, from which data in (a) were derived. Similar results were obtained in three independent experiments.

in suppression of eosinophilia. Infiltration of neutrophils, $\mathrm{T}$ cells, B cells, dendritic cells, and macrophages was also induced by allergen (Figure 4), and the numbers of these cells were also reduced. However these effects appeared to be dependent on the adjuvant, particularly where p 25 was included in the vaccine. By contrast, vaccine containing p 25 had no effect on any cellular infiltrates in HDM-sensitized animals, which showed very high levels of eosinophilia coupled with negligible neutrophilia when compared to OVA-sensitized animals. The data indicate that although Th1 adjuvants alone can reduce overall airway inflammation, the induction of allergen-specific CD8 T cells is more effective, particularly for suppression of eosinophil infiltration. Furthermore, such vaccines decrease total levels of lung inflammation rather than skewing from an eosinophilic toward a neutrophilic inflammatory profile.

\section{Vaccination enhances Th1 and inhibits Th2 cell responses in the airways}

We hypothesized that allergen-specific CD8 cells would regulate the Th1/Th2 balance in the lung. We determined Th1/Th2 phenotypes of BAL $T$ cells from mice vaccinated and challenged as in Figure 4. Th1 and Th2 cells were detectable in mice sensitized to OVA or HDM but then given PBS (Figure 5a). In OVA-sensitized mice, CASAC + p25 adjuvant alone increased Th1 polarization in BAL CD4 cells $(P<0.05)$, and decreased Th2 cells $(P<0.05)$. The addition of SIINFEKL led to significantly higher Th1 polarization when compared to CASAC + p 25 alone $(P<0.05)$, demonstrating that allergen-specific CD8 T cells promote the generation of Th1 effector cells. Although HDM induced a higher percentage of Th2 cells than OVA $(P<0.0005)$, vaccination with CASAC + p $25+$ SIINFEKL had no significant effect on the Th1/Th2 profile.

BAL CD8 T cells were less abundant than CD4 and were highly polarized toward a Tc1/IFN- $\gamma$ profile even in unsensitized animals (Figure 5a). Some Tc2-type, IL-5 positive CD8s were detectable in sensitized lungs and these were marginally inhibited by vaccination $(P<0.05)$. Typical flow cytometric profiles of cytokine staining from which the data in Figure 5a are derived are shown in Figure 5b.

\section{Effect of vaccination on BAL cytokines}

Levels of IFN- $\gamma$, IL-5, IL-13, and IL-17 were measured in BAL supernatants (Figure 6). Vaccination was found to significantly enhance BAL IFN- $\gamma$ levels $(P<0.05, n=6)$, although unlike the CD4 Th1 data this was independent of the presence of SIINFEKL. IL-13, not IL-5, was the major cytokine detectable in BAL postchallenge. However IL-13 was not affected by vaccination. Interestingly, despite the extremely low levels of IL-17 detectable in BAL fluid, CASAC significantly suppressed IL-17 levels. HDM induced high levels of all cytokines, which were unaffected by the vaccine.

\section{Suppression of airway hyperreactivity by vaccine-induced allergen-specific CD8 $\mathrm{T}$ cells}

Lastly, the effects of vaccination on airway hyperreactivity in response to increasing inhaled methacholine challenge are shown in Figure 7. Both OVA and HDM induced a similar degree of hyperreactivity to methacholine as demonstrated by increased resistance and decreased compliance of the lung. Only the complete vaccine containing SIINFEKL significantly prevented airway hyperreactivity $(P<0.05$ for resistance; $P<0.01$ for compliance; Figure 7 upper panels), although there was a trend toward decreased resistance with adjuvant alone. There was a clear and significant difference in the lung compliance data between adjuvant alone and adjuvant + SIINFEKL, demonstrating that SIINFEKL-specific CD8 T cells have a beneficial effect on lung function. Comparison of the data against groups of mice sensitized and challenged with HDM (Figure 7 lower panels) showed that the effect of the SIINFEKL vaccine was allergen specific, i.e., it had no effect on airway hyperreactivity induced by HDM. Thus the data confirm that targeting the MHC class Irestricted CD8 T-cell response is able to inhibit the key features of allergic airway disease in this model.

\section{DISCUSSION}

The opposing effects of CD4 and CD8 T cells on allergic immunopathology are related to the differential regulation of cytokine profiles within these subsets. Specifically, CD8 T cells retain a "type 1" cytokine profile, with copious IFN- $\gamma$ production, under most conditions, and are not dependent on IL-12 for acquisition 


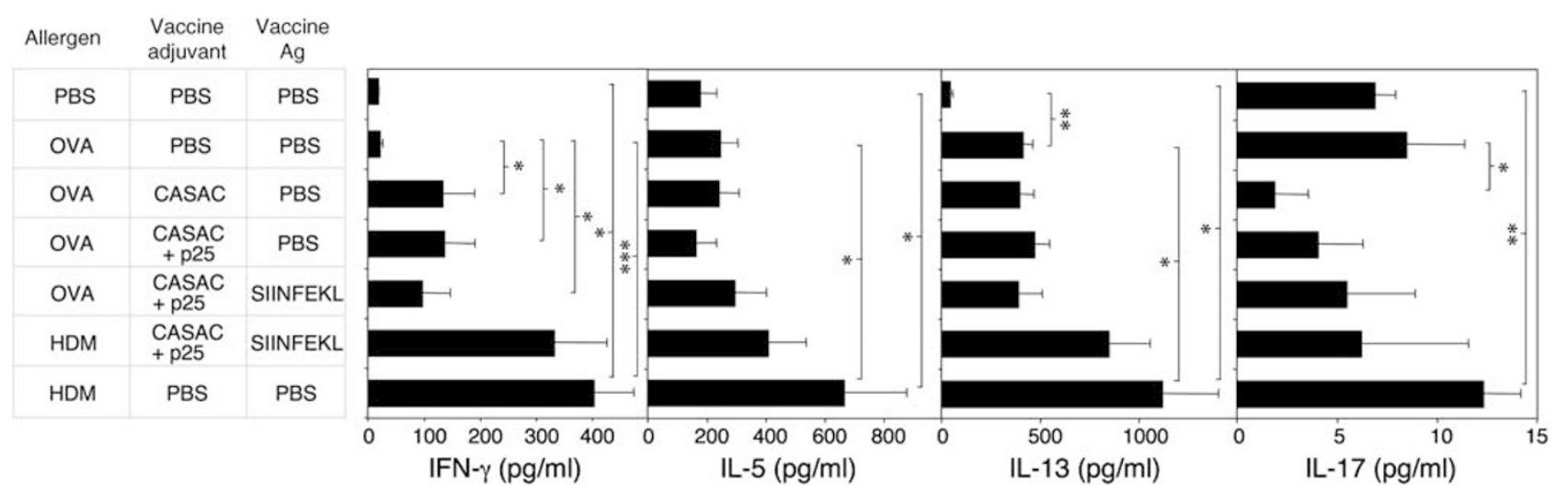

Figure 6 Vaccination alters the balance of Th1 and Th2 cytokines in BAL fluid. Levels of interferon- $\gamma$ (IFN- $\gamma$ ), interleukin-13 (IL-13), IL-5, and IL-17 were measured in the BAL fluid of mice on day 34 after repeated allergen challenge as in Figures 4 and $5 .{ }^{\star} P<0.05,{ }^{\star \star} P<0.005 ;{ }^{* \star \star} P<0.0005, n=6$. Similar results were obtained in three independent experiments.
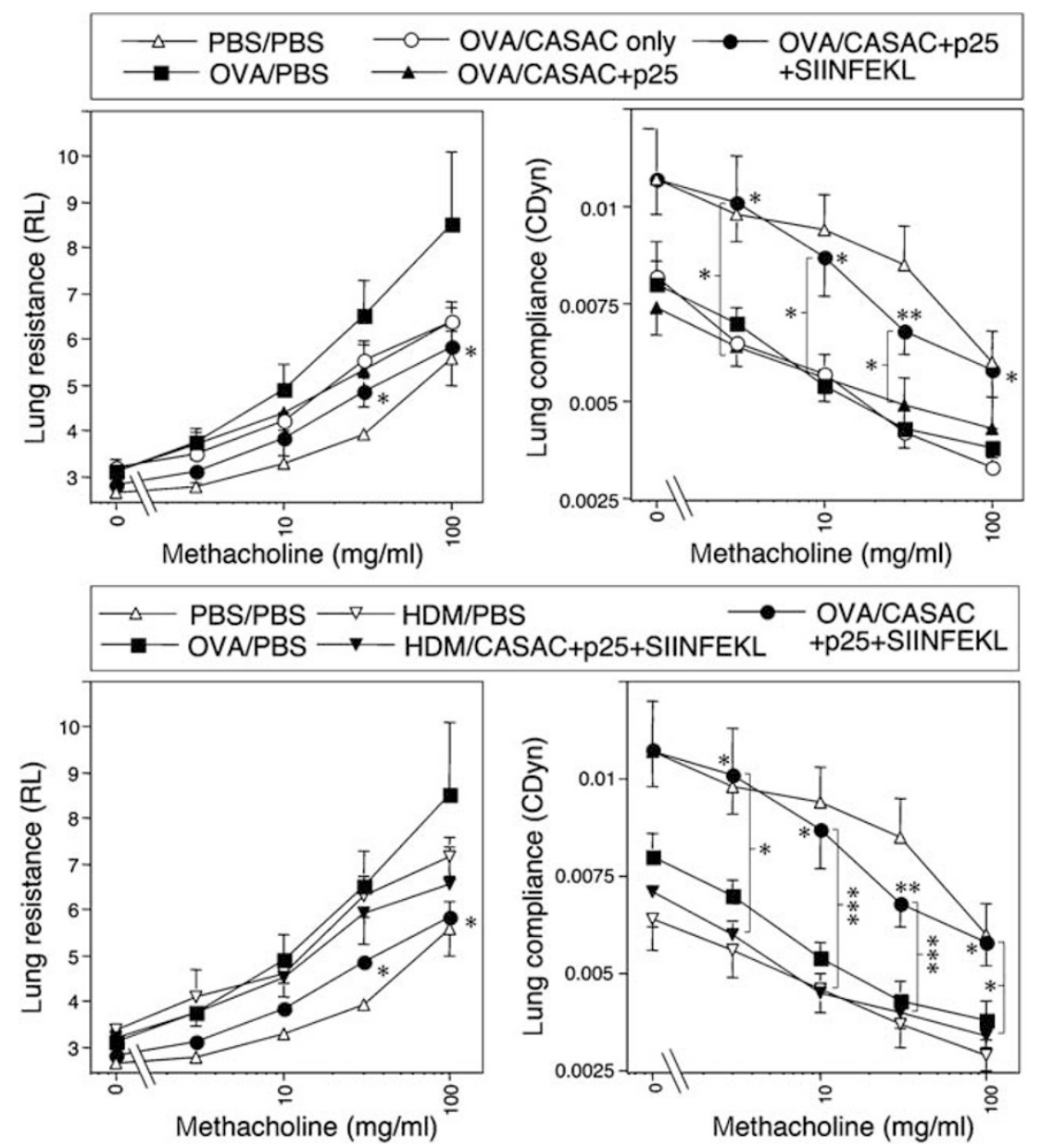

Figure 7 Suppression of airway hyperreactivity by allergen-specific CD8 T-cell responses to class I peptide. Lung resistance (left) and compliance (right) were measured in mice sensitized to OVA (upper panels) or HDM (lower panels) and challenged with various vaccine configurations as in Figures 4-6 Note: the same positive and negative control data in (upper panels) appear in (lower panels) alongside groups sensitized with HDM instead of OVA. Legends refer to "sensitizing allergen"/“vaccine components". ${ }^{\star} P<0.05,{ }^{\star \star} P<0.005,{ }^{\star \star \star} P<0.0005, n=6$.

of this function. ${ }^{21}$ By contrast, CD4 T cells are more likely to develop into Th2-polarized populations, which drive the allergic phenotype via a number of pathways. This is especially true in the neonatal period, when IFN- $\gamma$ production is deficient in CD4
T cells but much less so in CD8s. ${ }^{22}$ Allergen-specific CD8 T cells antagonize the $\mathrm{CD} 4 \mathrm{Th} 2$ response and suppress $\mathrm{IgE}$ responses, airway eosinophilia, and airway hyperreactivity. ${ }^{11,23-25}$ One mechanism for this regulatory pathway is the enhancement of 
Th1 development via induction of IL-12 from dendritic cells during CD8 cell interaction. ${ }^{14}$ Another mechanism is dependent on the propensity of CD8 $\mathrm{T}$ cells to produce IFN- $\gamma$, a cytokine that directly counteracts airway hyperreactivity and inflammation. ${ }^{6}$ Airway CD8 cells were highly skewed toward IFN- $\gamma$ production even without sensitization and challenge (Figure 5). IFN- $\gamma$ kills mast cells ${ }^{26}$ and strongly inhibits development of Th17 cells. ${ }^{27}$ Consistent with this, we found that IFN- $\gamma$-inducing vaccines suppressed levels of lung IL-17, which is implicated as a proinflammatory mediator in asthma, ${ }^{28}$ and neutrophils, which are thought to be recruited by Th17 cells. Recently induction of IL-18 from dendritic cells has also been shown to be critical for CD8 T-cell-mediated suppression of IgE. ${ }^{29}$ We therefore hypothesized that a vaccine inducing potent allergen-specific CD8 T-cell responses would inhibit multiple aspects of allergic immunopathology via an array of complementary pathways.

To develop such a vaccine, we first developed a powerful adjuvant combination, which overcame the limitations of other adjuvants when inducing CD8 responses to exogenous, nonreplicating antigens. Although anti-CD40 antibody is known to have unique adjuvant properties, ${ }^{30}$ we previously showed that anti-CD40 could be replaced with a class II-restricted helper peptide to invoke a concomitant CD4 response. It is also not required when whole proteins containing class II epitopes are used as antigen. ${ }^{20}$ In the context of an anti-allergy vaccine, the helper peptide strategy would have the advantage of inducing a CD4 response that was not directed to allergen, but to an unrelated, preferably Th1-inducing antigen. In this study we chose a peptide derived from Mycobacterial Ag85. As shown here, the replacement of anti-CD40 with helper peptide also abolished the dramatic increase in both serum total IgE and IL-5 levels in BAL fluid seen with anti-CD40 exposure. Anti-CD40 can elicit B cell IgE synthesis in the absence of IL- $4^{31}$ and because $\mathrm{CD} 40$ is also expressed on eosinophils, ${ }^{32}$ it may have triggered IL-5 release from airway eosinophils. Clearly, such an antibody would not be desirable in human vaccines and a helper peptide approach would avoid indiscriminate immune activation. The helper peptide strategy, by targeting CD40 signals to the cognate interaction between $\mathrm{T}$ cells and dendritic cells, seemed to be particularly successful in enhancing Th1 responses in the lung. By eliciting a three-cell interaction between dendritic cells and allergen-reactive CD4 and CD8 cells in draining lymphoid tissue, IL-12/IL-18 secretion would be enhanced, thus deviating the immunopathologic Th2 response toward Th1. However, it is not clear from our data whether the lung Th1 cells induced by vaccination were allergen specific, because we observed significant effects using adjuvant alone. Although anti-CD40 + CASAC induces IL-12 from dendritic cells and is strongly Th1-skewing, ${ }^{20}$ it appears that it can also induce IL- 5 release. Its beneficial effect on lung function may have been due to high-level induction of IFN- $\gamma$ from non-CD4 cells in the lung. Anti-CD40 mediated activation of dendritic cells may have induced release of IFN- $\gamma$ directly or from NK/NKT cells via IL-12 secretion.

Our data suggest that MHC class I-restricted peptide vaccines would be more effective than existing immunotherapeutic strategies. Whole allergen and class II peptides have demonstrated clinical benefit. ${ }^{33}$ These strategies rely largely on induction of regulatory $\mathrm{T}$ cells, predominantly of a $\mathrm{CD} 4^{+} \mathrm{IL}-10^{+}$phenotype. ${ }^{34}$ Our strategy presented here differs in that deliberate vaccination, rather than tolerization, is used. By targeting the CD8 component of the allergen response, it appears possible to redress the dysregulated balance between Th1 and Th2 cytokines characteristic of allergic airway inflammation and to prevent $d e$ novo IgE sensitization. The induction of allergen-specific CD8 cells enhanced Th1 polarization of lung CD4 cells as revealed by restimulation, but had little effect on the concentrations of Th2 cytokines in BAL fluid. It is possible that the reduced numbers of inflammatory cells in vaccinated animals resulted in less cytokine consumption.

Although adoptive transfer of in vitro-generated allergen-specific Th1 cells induces damaging lung inflammation ${ }^{35}$ and can exacerbate subsequent Th2 responses, ${ }^{36}$ the immune deviation strategy appears to target the development of Th2 responses and is therefore able to block Th2 pathology. It may be that by inducing a de novo response with a vaccine, natural regulatory pathways are also induced which prevent the excessive lung inflammation seen with Th1 adoptive transfer. The strategy has the advantage of using a traditional adjuvanted vaccine approach, which would be expected to induce reliable and robust responses, as shown by the success of mass vaccination programs. Traditional immunotherapy, by contrast, relies on the induction of tolerance, which can be counteracted by inflammatory signals and requires long-term treatment. ${ }^{37} \mathrm{~A}$ further advantage is that Th1-skewing adjuvants, such as CASAC, would trigger Th1 promoting cytokines that could regulate responses to further allergens unrelated to the target allergen. This would be of great benefit in diseases involving multiple sensitizations, such as asthma. It might also address the limitation that few class I epitopes have been identified in common allergens, despite elegant work on the HLA-A0201-restricted epitope of Der p1 several years ago. ${ }^{38}$ Further work in animal models using complex allergens such as house dust mite would clearly be valuable to address this issue. Although adjuvant alone had some effect in our model, induction of allergen-specific CD8 cells was significantly more effective for improved lung function and reduced eosinophilia. Although treatment with whole allergen and Th1-skewing adjuvants such as CpG DNA are effective in the murine model, ${ }^{39}$ our data suggest that the more potently Th1-polarizing adjuvant combination we used, along with CD8 induction, could be more effective and perhaps long-lasting. It is as yet uncertain how closely the murine model used here reflects human disease, and whether chronic disease involving airway remodeling would be reversed by such an approach. In our study we used the vaccine after sensitization but before the first airway challenge, which does not reflect initiation of allergic asthma. It would be informative to test an equivalent vaccine in a mucosal sensitization model, thus avoiding possible confounding effects of alum in our system.

Vaccination failed to significantly inhibit long-lived, preestablished IgE responses induced by repetitive OVA/alum sensitization. This is not unexpected because IgE-secreting plasma cells are fully differentiated and therefore not susceptible to immune 
deviation; they are also extremely long-lived cells..$^{40}$ Long-term treatment with vaccines might eventually lead to IgE suppression as plasma cells reach the end of their life. Nevertheless, our data suggest that clinical use of such a vaccine would likely be most effective in monoatopic individuals treated early in life. As peptide therapy and the individual components of CASAC adjuvant have been tested safely in humans and we saw no additional neutrophilia, excessive Tc1 inflammation or other ill effects in vaccinated mice, we believe that the approach tested here has potential for translation toward clinical studies.

\section{METHODS}

Mice. C57BL/6 mice (6-8 weeks) from Harlan (Oxford, UK) were used. Protocols complied with UK Home Office regulations and were approved by our Animal Welfare Committee.

Antibodies and reagents. Anti-CD40 3/23 was from Serotec (Oxford, UK), other antibodies from BD (Oxford, UK) or Caltag-MedSystems (Towcester, UK). IL-17 enzyme-linked immunosorbent assay (ELISA) paired antibodies were from R\&D Systems (Oxford, UK). PE-H-2K ${ }^{\mathrm{b}}$ SIINFEKL Pro5 MHC Pentamer and SIINFEKL peptide were from ProImmune (Oxford, UK). Sigma Grade V OVA or HDM allergen (Greer Laboratories, Lenoir, NC) were absorbed to alum (Alu-Gel-S; Serva GmbH, Heidelberg, Germany) in PBS before injection.

Vaccines. $100 \mu \mathrm{g} \mathrm{OVA}_{257-264}$ (SIINFEKL) peptide per mouse was used as antigen to induce CD8 T-cell responses. This was combined with the novel CASAC adjuvant (Combined Adjuvant for Synergistic Activation of Cellular immunity) we have described elsewhere. ${ }^{20}$ The CASAC adjuvant consists of two synthetic Toll-like receptor agonists (here we used $25 \mu \mathrm{g}$ CpG 1826 DNA $+50 \mu$ g polyI:C) +100 ng IFN- $\gamma+0.2 \%$ Tween 80 in PBS, emulsified with $2 \%$ squalene oil. Except where whole OVA was used, either anti-CD40 or a class II helper peptide was added to CASAC. Anti-CD40 antibody $(3 / 23,25 \mu \mathrm{g})$ was added for direct signaling through CD40. The class II-restricted unrelated helper peptide antigen

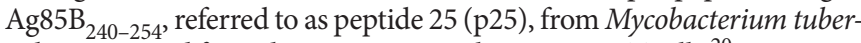
culosis was used for indirect CD40 signaling via CD4 T cells. ${ }^{20}$ SIINFEKL or whole OVA was mixed with adjuvant and vaccines were injected intradermally in two sites on the flanks $(2 \times 100 \mu \mathrm{l})$.

T-cell proliferation. Lymph node and spleen cells from SIINFEKL-vaccinated mice were taken 42 days after immunization and depleted of CD8 cells by autoMACS negative selection according to the manufacturer's instructions (Miltenyi Biotec, Wisley, UK), or left unfractionated. Cells were cultured at $5 \times 10^{6} / \mathrm{ml}$ in microwells with or without addition of $2 \mu \mathrm{g} / \mathrm{ml}$ SIINFEKL, and pulsed overnight with ${ }^{3} \mathrm{H}$-thymidine after 6 days for assessment of proliferation as described. ${ }^{13}$

Nascent and established IgE measurement. To determine the effect of vaccination on de novo IgE responses, mice were immunized i.d. with the vaccine combination indicated on days 0 and 10; total serum IgE was measured by ELISA on day 23 as described. ${ }^{41}$ Alternatively, mice were immunized i.d. with the vaccine combination indicated on day 0 , and then challenged i.p. with $400 \mu \mathrm{g}$ of OVA/alum on day 11 . Serum OVA-specific IgE was measured on days 11, 21, 28, 35, and 42, in arbitrary units by using an ELISA class-capture assay as described. ${ }^{42}$ To investigate the effect of vaccination on established IgE responses, mice were challenged i.p. on days 0,10 , and 21 with $400 \mu \mathrm{g}$ of OVA/alum, and immunized i.d. with the vaccine combination indicated on days 30 and 40. Mice were then rechallenged with $400 \mu \mathrm{g}$ of OVA/alum on day 54. Serum OVA-specific IgE was measured at the time points indicated.
Allergic airway disease model. Mice were sensitized to allergen with $10 \mu \mathrm{g}$ OVA/alum or $10 \mu \mathrm{g}$ HDM/alum i.p. on days 0 and 12 and immunized i.d. with PBS (control) or SIINFEKL peptide in combination with CASAC adjuvant combinations on day 20 . Blood was taken on day 28 to assess SIINFEKL-specific CD8 expansion in response to vaccination by pentamer staining (as described ${ }^{13}$ ). Mice were then challenged daily between days 28 and 33 with $50 \mu \mathrm{g}$ OVA (or $50 \mu \mathrm{g}$ HDM for HDM-sensitized mice) intranasally, and airway hyperreactivity was measured $24 \mathrm{~h}$ after last challenge, before collection of BAL by flushing lungs with $1 \mathrm{ml}$ PBS. Total leukocytes and eosinophils, neutrophils, T/B cells, and dendritic cells/macrophages were identified and counted by flow cytometry as described. ${ }^{13}$ Lung tissue T cells were prepared and analyzed, and BAL T-cell intracellular cytokine staining was performed, as described. ${ }^{13} \mathrm{IFN}$ $\gamma$, IL-5, IL-13, and IL-17 in BAL fluid were measured by ELISA using capture and detection antibody pairs, as described. ${ }^{14}$ Detection limits: IL-5 and IL-13-50 pg/ml; IFN- $\gamma-20 \mathrm{pg} / \mathrm{ml}$; IL-17-2.5 pg/ml.

Airway hyperreactivity measurements. Lung resistance and compliance ( RL and Cdyn) were measured in anaesthetized and tracheostomized mice in response to inhaled methacholine at concentrations of $3-100 \mathrm{mg} /$ $\mathrm{ml}$ as described previously. ${ }^{43}$

Data analysis. Experiments consisted of groups of mice $(n=4-6)$. All results are expressed as means \pm s.e.m. Unpaired Student's $t$-tests were used to compare groups except where deviation from normality was indicated, when Mann-Whitney tests were performed.

\section{ACKNOWLEDGMENTS}

We thank Sara Mathie and Karen Buckland for technical assistance.

\section{DISCLOSURE}

This work was funded by Asthma UK. KC is supported by the Medical Research Council. CML is supported by Wellcome Trust Senior Fellowship 057704. A patent application on CASAC adjuvant has been submitted by King's College London (inventors JWW \& AN); no other conflict of interest exists.

C) 2009 Society for Mucosal Immunology

\section{REFERENCES}

1. van der Pouw Kraan, T.C. et al. Reduced production of IL-12 and IL-12dependent IFN-gamma release in patients with allergic asthma. J. Immunol. 158, 5560-5565 (1997).

2. Holt, P.G. et al. Genetic "risk" for atopy is associated with delayed postnatal maturation of T-cell competence. Clin. Exp. Allergy 22, 10931099 (1992).

3. Baldini, M., Lohman, I.C., Halonen, M., Erickson, R.P., Holt, P.G. \& Martinez, F.D. A Polymorphism in the $5^{\prime}$ flanking region of the CD14 gene is associated with circulating soluble CD14 levels and with total serum immunoglobulin E. Am. J. Respir. Cell Mol. Biol. 20, 976-983 (1999).

4. Lauener, R.P. et al. Expression of CD14 and Toll-like receptor 2 in farmers' and non-farmers' children. Lancet 360, 465-466 (2002).

5. Upham, J.W. et al. Development of interleukin-12-producing capacity throughout childhood. Infect. Immun. 70, 6583-6588 (2002).

6. Yoshida, M. et al. Effect of interferon-gamma on allergic airway responses in interferon-gamma-deficient mice. Am. J. Respir. Crit. Care Med. 166, 451-456 (2002).

7. Kiwamoto, T. et al. Transcription factors T-bet and GATA-3 regulate development of airway remodeling. Am. J. Respir. Crit. Care Med. 174, 142-151 (2006).

8. Hashimoto, K. et al. Respiratory syncytial virus infection in the absence of STAT 1 results in airway dysfunction, airway mucus, and augmented IL-17 levels. J. Allergy Clin. Immunol. 116, 550-557 (2005).

9. Boguniewicz, M. et al. Treatment of steroid-dependent asthma with recombinant interferon- $\gamma$. Clin. Exp. Allergy 23, 785-790 (1993).

10. Martin, R.J. et al. The effects of inhaled interferon $\gamma$ in normal human airways. Am. Rev. Respir. Dis. 148, 1677-1682 (1993). 
11. Sedgwick, J.D. \& Holt, P.G. Induction of lgE-secreting cells and IgE isotype-specific suppressor $T$ cells in the respiratory lymph nodes of rats in response to antigen inhalation. Cell Immunol. 94, 182-194 (1985).

12. Isogai, S., Jedrzkiewicz, S., Taha, R., Hamid, Q. \& Martin, J.G. Resident $\mathrm{CD} 8^{+} \mathrm{T}$ cells suppress $\mathrm{CD} 4^{+} \mathrm{T}$ cell-dependent late allergic airway responses. J. Allergy Clin. Immunol. 115, 521-526 (2005).

13. Wells, J.W., Cowled, C.J., Giorgini, A., Kemeny, D.M. \& Noble, A. Regulation of allergic airway inflammation by class I-restricted allergen presentation and CD8 T-cell infiltration. J. Allergy Clin. Immunol. 119, 226-234 (2007).

14. Thomas, M.J., Noble, A., Sawicka, E., Askenase, P.W. \& Kemeny, D.M. CD8 T cells inhibit IgE via dendritic cell IL-12 induction that promotes Th1 T cell counter-regulation. J. Immunol. 168, 216-223 (2002).

15. Kalinski, P. \& Moser, M. Consensual immunity: success-driven development of T-helper-1 and T-helper-2 responses. Nat. Rev. Immunol. 5, 251-260 (2005).

16. Bratke, K. et al. Decrease of cytotoxic T cells in allergic asthma correlates with total serum immunglobulin E. Allergy 61, 1351-1357 (2006).

17. Polte, T. et al. CD137-mediated immunotherapy for allergic asthma. J. Clin. Invest. 116, 1025-1036 (2006).

18. Alexander, C., Tarzi, M., Larche, M. \& Kay, A.B. The effect of Fel d 1 derived T-cell peptides on upper and lower airway outcome measurements in cat-allergic subjects. Allergy 60, 1269-1274 (2005).

19. Hessel, E.M. et al. Immunostimulatory oligonucleotides block allergic airway inflammation by inhibiting Th2 cell activation and lgE-mediated cytokine induction. J. Exp. Med. 202, 1563-1573 (2005).

20. Wells, J.W., Cowled, C.J., Farzaneh, F. \& Noble, A. Combined triggering of dendritic cell receptors results in synergistic activation and potent cytotoxic immunity. J. Immunol. 181, 3422-3431 (2008).

21. Carter, L.L. \& Murphy, K.M. Lineage-specific requirement for signal transducer and activator of transcription (Stat) 4 in interferon- $\gamma$ production from CD4 ${ }^{+}$versus CD8 ${ }^{+}$T cells. J. Exp. Med. 189, 1355-1360 (1999).

22. White, G.P., Watt, P.M., Holt, B.J. \& Holt, P.G. Differential patterns of methylation of the IFN- $\gamma$ promoter at $\mathrm{CpG}$ and non-CpG sites underlie differences in IFN- $\gamma$ gene expression between human neonatal and adult CD45RO- T cells. J. Immunol. 168, 2820-2827 (2002).

23. Renz, H. et al. Inhibition of IgE production and normalization of airways responsiveness by sensitized CD8 T cells in a mouse model of allergeninduced sensitization. J. Immunol. 152, 351-360 (1994).

24. Holmes, B.J., MacAry, P.A., Noble, A. \& Kemeny, D.M. Antigen-specific CD8 ${ }^{+} \mathrm{T}$ cells inhibit immunoglobulin $\mathrm{E}$ responses and interleukin-4 production by CD4+ T cells. Eur. J. Immunol. 27, 2657-2665 (1997).

25. Hansen, G., Yeung, V.P., Berry, G., Umetsu, D.T. \& DeKruyff, R.H. Vaccination with heat-killed Listeria as adjuvant reverses established allergen-induced airway hyperreactivity and inflammation: role of $C D 8^{+} T$ cells and IL-18. J. Immunol. 164, 223-230 (2000).

26. Mann-Chandler, M.N. et al. IFN- $\gamma$ induces apoptosis in developing mast cells. J. Immunol. 175, 3000-3005 (2005).
27. Harrington, L.E. et al. Interleukin 17-producing CD4+ effector T cells develop via a lineage distinct from the Thelper type 1 and 2 lineages. Nat. Immunol. 6, 1123-1132 (2005).

28. Molet, S. et al. IL-17 is increased in asthmatic airways and induces human bronchial fibroblasts to produce cytokines. J. Allergy Clin. Immunol. 108, 430-438 (2001).

29. Salagianni, M., Loon, W.K., Thomas, M.J., Noble, A. \& Kemeny, D.M. An essential role for IL-18 in CD8 T cell-mediated suppression of IgE responses. J. Immunol. 178, 4771-4778 (2007).

30. Hawiger, D. et al. Dendritic cells induce peripheral $T$ cell unresponsiveness under steady state conditions in vivo. J. Exp. Med. 194, 769-779 (2001).

31. Ferlin, W.G. et al. CD40 signaling induces interleukin-4-independent lgE switching in vivo. Eur. J. Immunol. 26, 2911-2915 (1996).

32. Bureau, F. et al. CD40 engagement enhances eosinophil survival through induction of cellular inhibitor of apoptosis protein 2 expression: Possible involvement in allergic inflammation. J. Allergy Clin. Immunol. 110, 443-449 (2002).

33. Abramson, M.J., Puy, R.M. \& Weiner, J.M. Is allergen immunotherapy effective in asthma? A meta-analysis of randomized controlled trials. Am. J. Respir. Crit. Care Med. 151, 969-974 (1995).

34. Larche, M. Immunoregulation by targeting $T$ cells in the treatment of allergy and asthma. Curr. Opin. Immunol. 18, 745-750 (2006).

35. Hansen, G., Berry, G., DeKruyff, R.H. \& Umetsu, D.T. Allergen-specific Th1 cells fail to counterbalance Th2 cell-induced airway hyperreactivity but cause severe airway inflammation. J. Clin. Invest. 103, 175-183 (1999).

36. Randolph, D.A., Carruthers, C.J., Szabo, S.J., Murphy, K.M. \& Chaplin, D.D. Modulation of airway inflammation by passive transfer of allergenspecific Th1 and Th2 cells in a mouse model of asthma. J. Immunol. 162, 2375-2383 (1999).

37. Calderon, M.A., Birk, A.O., Andersen, J.S. \& Durham, S.R. Prolonged preseasonal treatment phase with Grazax sublingual immunotherapy increases clinical efficacy. Allergy 62, 958-961 (2007).

38. Seneviratne, S.L. et al. Allergen-specific $\mathrm{CD} 8^{+} \mathrm{T}$ cells and atopic disease. J. Clin. Invest 110, 1283-1291 (2002).

39. Broide, D. et al. Immunostimulatory DNA sequences inhibit IL-5, eosinophilic inflammation, and airway hyperresponsiveness in mice. J. Immunol. 161, 7054-7062 (1998).

40. Xiang, Z. et al. Fc $\gamma$ Rllb controls bone marrow plasma cell persistence and apoptosis. Nat. Immunol. 8, 419-429 (2007).

41. Noble, A., Leggat, J.A. \& Inderberg, E.M. CD8 ${ }^{+}$immunoregulatory cells in the graft-versus-host reaction: CD8 T cells activate dendritic cells to secrete interleukin-12/interleukin-18 and induce Thelper 1 autoantibody. Immunology 109, 476-486 (2003).

42. Eynott, P.R. et al. Role of nitric oxide in chronic allergen-induced airway cell proliferation and inflammation. J. Pharmacol. Exp. Ther. 304, 22-29 (2003).

43. Kearley, J., Barker, J.E., Robinson, D.S. \& Lloyd, C.M. Resolution of airway inflammation and hyperreactivity after in vivo transfer of $\mathrm{CD} 4{ }^{+} \mathrm{CD} 25^{+}$regulatory T cells is interleukin 10 dependent. J. Exp. Med. 202, 1539-1547 (2005). 